\title{
Management of adverse events related to checkpoint inhibition therapy
}

\author{
Jakob Daniel Rudzki
}

Received: 8 April 2018 / Accepted: 17 May 2018 / Published online: 12 June 2018

(C) The Author(s) 2018

\begin{abstract}
Summary IO treatments (immuno-oncology treatments) have become reality and are now daily practice or, in some cases, a daily challenge. New recommendations are being made with the prime purpose of increasing alertness and awareness as well as emphasizing standard operating strategies to deal with immune-related adverse events (ir-AEs) in patients treated with immune checkpoint inhibitors (ICI). This brief review refers to systemic reviews, guidelines and meta-analyses, randomized controlled trials and case series published from 2000 to the present. Existing recommendations for optimal management of toxicities vary according to organ systems affected and grading. Grade 1 toxicities (exception to the rule: neurologic, hematologic, cardiac manifestation) require close monitoring. Grade 2 toxicities prompt immediate treatment interruption combined with corticosteroid administration (prednisone or methylprednisolone $0.5-1 \mathrm{mg} / \mathrm{kg} /$ day) until the symptoms revert to grade 1 or less. ir-AEs up to grade 3 or 4 justify suspension of treatment together with increased dosage of prednisone or methylprednisolone $(1-2 \mathrm{mg} / \mathrm{kg} /$ day $)$ combined with close monitoring to continuously adapt the current immunosuppressive strategy. In some cases, a different additional immunosuppressive agent has to be evaluated. Only when all symptoms have disappeared and immunosuppressive treatment produces a response can all immunosuppressive agents be tapered. Endocrinopathies are the exception to the rule and are
\end{abstract}

OA Dr. J. D. Rudzki

Medical University Innsbruck, Innsbruck, Austria

OA Dr. J. D. Rudzki ( $\varangle)$

UKIM V-Tirol Kliniken, Landeskrankenhaus Innsbruck, Anichstraße 35, 6020 Innsbruck, Austria

jakob.rudzki@tirol-kliniken.at mostly controllable by hormone replacement, at least in low-grade manifestation. This short review focuses on the main aspects that help manage immune-related side-effects and elucidates all the additional aspects surrounding and contributing to successful treatment and management of cancer patients.

Keywords Immunotherapy - Checkpoint inhibition • Immune-related adverse events · Guidelines · Recommendations

\section{Immunotherapy and immune-related adverse} events

The rise of the checkpoint blockade has been celebrated as the breakthrough of the year 2011. There is a wide diversity of checkpoint-inhibiting antibodies: anti-CTLA-4 (cytotoxic T-lymphocyte-associated Protein 4; Yervoy ${ }^{\circledR}$ ipilimumab [1]), anti-PD-1 (programmed cell death Protein 1; Opdivo ${ }^{\circledR}$ nivolumab [2, 3]; Keytruda ${ }^{\circledR}$ pembrolizumab [4]) or anti-PDL1 directed against the ligand of PD-1 (programmed death ligand 1; Bavencio ${ }^{\circledR}$ avelumab [5], Tecentriq ${ }^{\circledR}$ atezolizumab [6], Imfinzi ${ }^{\circledR}$ durvalumab [7]), all of which received approval from the US Food and Drug Administration (FDA) and some from the European Medicines Agency (EMA) for treatment of diverse entities of malignancies (metastatic melanoma, metastatic Merkel cell carcinoma, squamous and nonsquamous lung cancer, bladder cancer, metastatic renal cell cancer, therapy-resistant Hodgkin's lymphoma, head and neck cancer, hepatic cell cancer, mismatch repair-deficient/microsatellite instabilityhigh solid tumors). While these have to be mentioned, the brevity of this overview precludes their discussion.

Treatment of metastatic melanoma and both entities of lung cancer (squamous and nonsquamous cell carcinoma) mainly contributed to the success of 
ICIs as part of current treatment protocols. While in many cases sustained antitumor response directly correlates with clinical benefit, it may be associated with a wide spectrum of adverse events (presented at the American Association for Cancer Research 2017 Annual Meeting in Washington and commented by Julie Brahmer: Abstract No.: CT077, on 3 April 2017; Checkmate 209-003 phlb study: 5a OS for the overall study population: $16 \%, 95 \%$ CI 10-23. Survival was seen across PD-L1 expression levels and tumor histologies, but with the bias of not being randomized, blinded or controlled). The reported AEs mainly differ from all known side-effects generated by cytotoxic therapies (chemotherapy, radiotherapy) or antibodies or combinations of them. A wide range of organ systems can be affected (skin, gastrointestinal tract, lungs, endocrine, nervous: peripheral as well as central, ocular, cardiovascular system). The occurring inflammatory reactions strongly resemble autoimmune disorders. While severity is generally mild, even lifethreatening complications have been detected. There are some differences between anti-CTLA-4 and antiPD1/-PDL1 antibodies, especially in grading and duration of occurrence of ir-AEs $[8,9]$. There is no direct correlation to drug administration, with infusion related reactions (IRR) documented in very rare cases, namely fewer than $1 \%$ of patients. ir-AEs occur even after treatment has been suspended for weeks or even months and this has to be kept in mind when managing patients receiving IO (immuno-oncology) treatment [8].

\section{Recognition is the first step to successful treatment}

Many very interesting reviews provide extensive information and elaborate this topic in a concise manner [10-12].

Successful management of ir-AEs irrespective of the affected organ systems demands that the patient, family caregivers and the patient's GP (general practitioner) be kept abreast of the latest IO developments. All of these persons need to be informed about the IO concept and the clinical profile of possible AEs before the concept is initiated, and their information level should be maintained throughout the treatment period. The aim is to generate a "sound" but high level of suspicion for the fact that new symptoms occurring during and even after treatment start may be primarily related to ICI treatment. Grading of toxicities follows CTCAE version 5.0 (National Cancer Institute: Common Terminology Criteria for Adverse Events [CTCAE] 5.0.).

\section{Recommended management of ir-AEs}

An increasing number of treatment approaches using ICIs require early recognition of symptoms in connection with changes in laboratory results. Every new symptom must be viewed in connection with the treatment and regarded as an ir-AE until proven otherwise. Diagnostic tools (laboratory results, body scans) are helpful in excluding ir-AEs. Identifying patients at higher risk for ir-AEs demands prompt and aggressive reaction. Recent data suggest that patients suffering from autoimmune diseases are eligible for checkpoint inhibition although they are currently excluded from all trials. There are ways to treat them safely while remaining more alert for unexpected adverse events, although they are still seriously underrepresented [13] and could form a group of high-risk patients. Indeed, such patients do not show a higher incidence of ir-AEs despite having active disease. Hence, treatment-specific risk factors are defined by IO/IO combinations and by the checkpoint inhibitor itself due to differences in incidence and severity of ir-AEs between anti-CTLA-4 [1], anti-PD1 [2-4], and antiPDL1 antibodies [6, 7].

A heightened awareness for early recognition and treatment helps to mitigate the severity of ir-AEs.

\section{Organ-specific recommendations for ir-AEs}

In most cases (with some exceptions) ir-AEs grade 2 can be managed by promptly interrupting treatment and providing supportive care. Questionnaires or standardized assessments assist in early recognition. New symptoms or changes in a patient's health indicate early onset of ir-AEs. Giving patients wallet cards containing detailed information about symptoms and the patient's immunotherapy history (documented ir-AEs with grading) ensures that all care providers (GPs but also emergency department staff) have additional information to handle the situation and avoid morbidity and ultimately even mortality.

In the case of grade 1 toxicities, watchful continuation of the IO treatment is generally appropriate. However, without complete resolution of symptoms or deterioration to grade 2 or worse immunosuppressive treatment must be started at 0.5 to $1 \mathrm{mg} / \mathrm{kg} /$ day prednisone or equivalent corticosteroid combined with tapered discontinuation of treatment. Such tapering of systemic corticosteroids over 4-6 weeks is performed contingent on resolution of the individual's immunerelated reaction. Grade 3 or 4 ir-AEs involve some substantial differences concerning the affected organ system. These differences are discussed in the subsequent part of this article and summarized in Table 1 (pointing out the management of severe adverse events $=$ CTCAE $\geq$ grade 3 in defined organ toxicities).

\section{Skin toxicities}

In the case of failure of high-dose corticosteroid treatment (up to $2 \mathrm{mg} / \mathrm{kg} /$ day) an additional immunosuppressive therapy regimen is available: rituximab $375 \mathrm{mg} / \mathrm{m}^{2}$ is recommended instead of high-dose corticosteroids for bullous dermatoses and cyclosporine 
Table 1 Management of ir-AEs in patients treated with ICls/ overview of the most relevant (S)AEs (severe adverse events) focused on severe adverse events (CTCAE 5.0 $\geq 3$ ). (Adapted from [10]; based on expert consensus based recommendations with benefits outweighing harms-strength of recommendations is moderate)

1. Skin toxicities
1.1. Rash/
inflammatory
dermatitis
Erythema multiforme minor,
lichenoid, eczematous

\subsection{Bullous}

\section{dermatoses}

including bullous pemphigoid or other autoimmune bullous dermatoses, bullous drug reaction

1.3. SCARs (including SJS, TEN, acute generalized exanthematous pustulosis, DRESS/ DIHS)

severe changes in either structure or function of skin, the appendages or the mucous membranes due to a drug

\section{Grading}

According to CTCAE is a challenge for skin. Severity is based on BSA tolerability, morbidity and duration

G3: as G2 but with failure to respond to indicated interventions for G2 dermatitis

G4: all severe rashes unmanageable with prior interventions

G3: skin sloughing covering $>30 \%$ BSA with associated pain and limiting self-care ADL

G4: blisters covering $>30 \%$ BSA with associated fluid or electrolyte abnormalities

G3: skin sloughing covering $<10 \%$ BSA with mucosal involvement associated signs (e.g., erythema, purpura, epidermal detachment, mucous membrane detachment

G4:skin erythema \& blistering/ sloughing covering $\geq 10$ to $>30 \%$ BSA with associated signs (e.g., erythema, purpura, epidermal detachment, mucous membrane detachment) and/or systemic symptoms and concerning associated blood work abnormalities (e.g., liver function test elevations in the setting of DRESS/DIHS)

\section{Management}

Holding ICI \& weekly monitoring and treat with topical emollients, antihistamines \& high-potency topical corticosteroids + initiate $1-2 \mathrm{mg} / \mathrm{kg}$ corticosteroid ${ }^{\mathrm{a}}+$ tapering over at least 4 weeks

Systemic corticosteroids IV a $1-2 \mathrm{mg} / \mathrm{kg}$ with slow tapering when toxicity resolves. Consult with dermatology. Consider alternative antineoplastic therapy, if ICls are the patient's only option, consider restarting once these adverse effects have resolved to a G1 level

Hold ICI \& consult with dermatology; administer IV corticosteroids ${ }^{\mathrm{a}} 1-2 \mathrm{mg} / \mathrm{kg}$, tapering over at least 4 weeks, if bullous pemphigoid is diagnosed, it may be possible to avoid long term use of systemic corticosteroids and start with rituximab as an alternative approach to treat ir-AEs. Seek infectious disease consultation if patient have secondary cellulitis or other infection risk factors like neutropenia etc.

Permanently discontinue ICls, place patient under supervision of a dermatologist, administer IV corticosteroids ${ }^{\mathrm{a}} 1-2 \mathrm{mg} / \mathrm{kg}$ \& treat with rituximab as an alternative approach + seek infectious disease consultation if patient have secondary cellulitis or other infection risk factors like neutropenia etc.

Hold ICI \& consult with dermatology; treat skin with topical emollients \& other petrolatum emollients; administer IV corticosteroids ${ }^{\mathrm{a}} 0.5-1 \mathrm{mg} / \mathrm{kg}$ \& convert to oral on response, wean over at least 4 weeks; add additional immunosuppressive agent (cyclosporine) in corticosteroid unresponsive cases

Permanently discontinue ICls; admit patient immediately to a burn unit or ICU with consulted dermatology \& wound care services. Initiate IV corticosteroids ${ }^{\mathrm{a}} 1-2 \mathrm{mg} / \mathrm{kg}$ and tapering when toxicity resolves to normal; IVIG or cyclosporine may also be considered in corticosteroid unresponsive cases
G3: increase of 7 or more stools per day over baseline, incontinence, hospitalization indicated, severe increase in ostomy output compared with baseline, limited self-care ADL

G4: life-threatening situation; urgent intervention indicated

\section{Hepatitis}

G3: symptomatic liver dysfunction, fibrosis by biopsy, compensated cirrhosis, reactivation of chronic hepatitis (AST or ALT 5-20 $\times$ ULN and/or total bilirubin $3-10 \times$ ULN)

G4: decompensated liver function (e.g., ascites, coagulopathy, encephalopathy, coma; AST or ALT $>20 \times$ ULN and/or total bilirubin $>10$ ULN) more urgent endoscopy) \& calprotectin (to follow-up on disease activity); screening laboratories (HIV, hepatitis $A \&$ \&, blood quantiferon for tuberculosis) to prepare patients for infliximab treatment \& should be routinely determined in patients at high risk; imaging (e.g., CT scan of abdomen \& pelvis \& Gl endoscopy with biopsy) for early detection of ulceration in the colon that can predict a corticosteroid-refractory course that may require early infliximab treatment

Consider permanently discontinuing CTLA-4 agents and may restart PD-1, PD-L1 agents if patient can recover to G1 or less., administer corticosteroids ${ }^{\mathrm{a}}$ $1-2 \mathrm{mg} / \mathrm{kg}$; if symptoms persist $\geq 3-5$ days or recur after improvement, consider administering IV corticosteroids ${ }^{\mathrm{a}}$ or infliximab; consider colonoscopy in cases where patients have been on immunosuppression \& may be at risk for opportunistic infections (i.e., CMV colitis) \& for those who are anti-TNF or corticosteroid refractory

Permanently discontinue treatment; administer $1-2 \mathrm{mg} / \mathrm{kg}$ corticosteroids ${ }^{\mathrm{a}}$ until symptoms improve to G1 \& then start taper over 4-6 weeks; if symptoms are refractory to corticosteroids administer infliximab $5-10 \mathrm{mg} / \mathrm{kg}$ within $2-3$ days. If there is concern of new infection or symptoms remain refractory to treatment consider lower $\mathrm{Gl}$ endoscopy and consider vedolizumab in patients refractory to infliximab and/or contraindicated to TNF-alpha blockade. ${ }^{\text {b }}$

Permanently discontinue ICls, immediately start corticosteroid ${ }^{\mathrm{a}} 1-2 \mathrm{mg} / \mathrm{kg}$; if no improvement assessed after 3 days or if corticosteroid refractory, consider mycophenolate mofetil or azathioprine, daily laboratories \& close monitoring for patients with AST/ALT $>8 \times$ ULN and or elevated TB $3 \times$ ULN (infliximab should not be used - potential risk of liver failure); corticosteroid taper can be attempted around 4-6 weeks; re-escalate if needed, optimal duration is unclear

Permanently discontinue ICls, immediately start corticosteroid $2 \mathrm{mg} / \mathrm{kg}$, if no improvement assessed after 3 days or if corticosteroid refractory the same management as in the case of G3 
Table 1 (Continued)

\begin{tabular}{|c|}
\hline $\begin{array}{l}\text { 4. Pneumonitis } \\
\text { focal or diffuse inflammation } \\
\text { of the lung parenchyma } \\
\text { (typically identified on } C T \\
\text { imaging) }\end{array}$ \\
\hline 5. Endocrinopathies \\
\hline $\begin{array}{l}\text { 5.1. Primary hypothy- } \\
\text { roidism }\end{array}$ \\
\hline
\end{tabular}

5.2. Hyperthyroidism

\subsection{Primary adrenal insufficiency}

\subsection{Hypophysitis}

Low ACTH with low cortisol; low or normal TSH with a low

FT4; hypernatremia and volume depletion with diabetes insipidus; low testosterone or estradiol with low LH \& FSH

\subsection{Diabetes}

T1DM (autoimmune) results from islet destruction and is often acute onset, with ketosis and an insulin requirement

\section{Grading}

G3: severe symptoms, hospitalization required, involves all lung lobes or $>50 \%$ of lung parenchyma, limiting self-care ADL, oxygen indicated

G4: life threatening respiratory compromise, urgent intervention indicated (intubation)

G3-4: severe symptoms, medically significant or life-threatening consequences, unable to perform $A D L$

G3-4: severe symptoms, medically significant or life-threatening consequences, unable to perform ADL

G3-4: severe symptoms, medically significant or life-threatening consequences, unable to perform ADL

G3-4: severe symptoms, medically significant or life-threatening consequences, unable to perform ADL

G3-4: severe symptoms, medically significant or life-threatening consequences, unable to perform ADL G3: > 250-500 mg/d

G4: $>500 \mathrm{mg} / \mathrm{dl}$

\section{Nervous system toxicities}

\subsection{Myastenia gravis}

\subsection{Guillain-Barré} syndrome

Corticosteroids are usually not recommended for idiopathic Guillain-Barré syndrome, however, in ICls related forms a treatment is reasonable

\subsection{Peripheral neu-} ropathy

\subsection{Autonomic neu- ropathy}

6.5. Aseptic meningitis

\subsection{Encephalitis}

G3-4: limiting self-care and aids warranted, weakness limiting walking, any dysphagia, facial weakness, respiratory muscle weakness or rapidly progressive symptoms

G3-4: severe, limiting self-care and aids warranted, weakness limiting walking, any dysphagia, facial weakness, respiratory muscle weakness or rapidly progressive symptoms

G3-4: severe, limiting self-care and aids warranted, weakness limiting walking; severe may be Guillain-Barré syndrome

G3-4: severe limiting self-care and aids warranted

G3-4: severe, limiting self-care and aids warranted

G3-4: severe, limiting self-care and aids warranted

\section{Management}

Permanently discontinue ICl, empirical antibiotics; administer corticosteroid ${ }^{\mathrm{a}}$ $1-2 \mathrm{mg} / \mathrm{kg}$, no improvement after $48 \mathrm{~h}$, add infliximab $5 \mathrm{mg} / \mathrm{kg}$ or mycophenolate mofetil IV $1 \mathrm{~g}$ twice a day or IVIG for 5 days or cyclophosphamide; taper corticosteroids over 4-6 weeks. Pulmonary \& infectious disease consults if necessary,

$B A L \pm$ transbronchial biopsy offered

Hold ICI until symptoms resolve to baseline with appropriate supplementation; endocrine consultation, add IV therapy if signs of myxedema (bradycardia, hypothermia); thyroid supplementation and reassessment as in G2

Hold ICls until symptoms resolve to baseline, endocrine consultation, beta blocker for symptomatic relief, for severe symptoms administer corticosteroids ${ }^{\mathrm{a}} 1-2 \mathrm{mg} / \mathrm{kg}$ and taper over 1-2 weeks \& consider also use of SSKI or thionamide (methimazole or PTU)

Hold ICls until patient is stable on replacement hormone, endocrine consultation, IV stress dose corticosteroids on presentation (hydrocortisone $100 \mathrm{mg}$ or dexamethasone $4 \mathrm{mg}$ - if diagnosis is not clear \& stimulation testing will be needed). Taper stress dose corticosteroids down to maintenance doses over 7-14 days after discharge, continue maintenance therapy as in G1 and titrate down as symptoms dictate

Consider MRI of the brain with or without contrast with pituitary/sellar cuts in patients with multiple endocrine abnormalities \pm new severe headaches or complaints of vision changes

Hold ICls until patient is stable on replacement hormone; hormonal replacement as in G1; consider initial pulse therapy with corticosteroid ${ }^{\mathrm{a}} 1-2 \mathrm{mg} / \mathrm{kg}$ oral daily with subsequent tapering over at least $1-2$ weeks

Hold ICls until glucose control is obtained on therapy with reduction of toxicity to G1 or less, urgent endocrine consultation for all patients, admit for inpatient management: possibility of developing DKA

Permanently discontinue ICls; admit patient, may need ICU level monitoring, neurology consult, continue corticosteroids ${ }^{\mathrm{a}} 1-2 \mathrm{mg} / \mathrm{kg}$ and initiate IVIG $2 \mathrm{~g} / \mathrm{kg}$ IV over 5 days $(0.4 \mathrm{~g} / \mathrm{kg} /$ day) or plasmapheresis for 5 days, frequent pulmonary function assessment, daily neurologic review. Pyridostigmine starting at $30 \mathrm{mg}$ orally three times a day and gradually increase based on symptoms

Rapid transfer to ICU-level monitoring, start IVIG ( $0.4 \mathrm{~g} / \mathrm{kg} /$ day for 5 days) or plasmapheresis; administer corticosteroids ${ }^{\mathrm{a}} 2-4 \mathrm{mg} / \mathrm{kg} /$ day followed by slow taper, frequent pulmonary function assessment, daily neurologic review; nonopioid management of neuropathic pain

Proceed as per Guillain-Barré syndrome

Permanently discontinue ICls; admit patient; initiate corticosteroid ${ }^{\mathrm{a}} 1 \mathrm{~g}$ daily for 3 days followed by taper, neurologic consultation

Hold ICl and discuss resumption with patient only after taking into account the risks and benefits.

Consider empirical antiviral (IV acyclovir) and antibacterial therapy until CSF results-once bacterial \& viral infection are negative, may closely monitor off corticosteroids $^{\mathrm{a}}$ or consider oral corticosteroids ${ }^{\mathrm{a}} 0.5-1 \mathrm{mg} / \mathrm{kg} /$ day if moderate/severe symptoms

Proceed as per aseptic meningitis, consider administration of corticosteroida $1-2 \mathrm{mg} / \mathrm{kg}$ if severe: pulse corticosteroid ${ }^{\mathrm{a}} 1 \mathrm{~g}$ IV for $3-5$ days plus IVIG $2 \mathrm{~g} / \mathrm{kg}$ over 5 days;

if positive for autoimmune encephalopathy antibody and limited or no improvement, consider rituximab or plasmapheresis in consultation with neurology 
Table 1 (Continued)

\begin{tabular}{|c|c|c|}
\hline & Grading & Management \\
\hline $\begin{array}{l}\text { 6.7. Transverse myeli- } \\
\text { tis }\end{array}$ & $\begin{array}{l}\text { G3-4: severe, limiting self-care and } \\
\text { aids warranted }\end{array}$ & $\begin{array}{l}\text { Permanently discontinue ICls; administer corticosteroid }{ }^{\mathrm{a}} 2 \mathrm{mg} / \mathrm{kg} \text {; } \\
\text { strongly consider higher doses of } 2 \mathrm{~g} / \text { day for } 3-5 \text { days; strongly consider IVIG }\end{array}$ \\
\hline \multirow{2}{*}{$\begin{array}{l}\text { 7. Myocarditis, } \\
\text { pericarditis, } \\
\text { arrhythmias, impaired } \\
\text { ventricular function } \\
\text { with heart failure and } \\
\text { vasculitis }\end{array}$} & $\begin{array}{l}\text { G3: moderately abnormal testing or } \\
\text { symptoms with mild activity }\end{array}$ & Hold ICls and permanently discontinue after G1 \\
\hline & $\begin{array}{l}\text { G4: moderate or severe decompen- } \\
\text { sation; IV medication or intervention } \\
\text { required, life-threatening conditions }\end{array}$ & $\begin{array}{l}\text { High dose corticosteroids } \mathrm{s}^{\mathrm{a}} 1-2 \mathrm{mg} / \mathrm{kg} \text { initiated rapidly (oral or IV depending on symp- } \\
\text { toms), admit patient, cardiology consultation; in patients without immediate response } \\
\text { to high dose corticosteroids }{ }^{\mathrm{a}} \text {, increase to } 1 \mathrm{~g} \text { every day of corticosteroids and consider } \\
\text { the addition of either mycophenolate, infliximab or antithymocyte globulin }\end{array}$ \\
\hline \multicolumn{3}{|c|}{ Statement: Holding of checkpoint inhibitor therapies recommended for all grades of cardiovascular complications } \\
\hline \multirow[t]{2}{*}{ 8. Nephritis } & $\begin{array}{l}\text { G3: creatinine }>3 \times \text { baseline } \\
\text { or }>4.0 \mathrm{mg} / \mathrm{dl} \text {; hospitalization indi- } \\
\text { cated }\end{array}$ & $\begin{array}{l}\text { Permanently discontinue ICls; administer corticosteroids }{ }^{\mathrm{a}} 0.5-1 \mathrm{mg} / \mathrm{kg} \text {, if worsening or } \\
\text { no improvement increase to } 1-2 \mathrm{mg} / \mathrm{kg} / \text { day and taper over } 4-6 \text { weeks }\end{array}$ \\
\hline & $\begin{array}{l}\text { G4: life threatening consequences; } \\
\text { dialysis indicated }\end{array}$ & $\begin{array}{l}\text { Consult nephrology, evaluate for other causes; administer corticosteroids }{ }^{a} \\
1-2 \mathrm{mg} / \mathrm{kg} / \text { day }\end{array}$ \\
\hline \multicolumn{3}{|c|}{$\begin{array}{l}\text { ACTH adrenocorticotropic hormone, } A D L \text { activities of daily living, } A L T \text { alanine aminotransferase, } A S T \text { aspartate aminotransferase, } B A L \text { bronchoalveolar lavage, } \\
B S A \text { body surface area, CSF cerebrospinal fluid, CTCAE } 5.0 \text { Common Terminology Criteria for Adverse Events, CTLA-4 cytotoxic T-lymphocyte-associated } \\
\text { Protein } 4, D I H S \text { drug-induced hypersensitivity syndrome, DKA diabetic ketoacidosis, DRESS drug reaction with eosinophilia and systemic syndrome, FT4 free } \\
\text { thyroxine, G grade, HIV humane immunodeficiency virus, ICls immune-checkpoint inhibitors, ICU intensive care unit, IV intravenous, IVIG intravenous } \\
\text { immunoglobulin, } M R I \text { magnetic resonance imaging, } P D-1 \text { programmed cell death Protein } 1, P D-L 1 \text { programmed death ligand } 1, P T U \text { propylthiouracil, } \\
\text { SCARs severe cutaneous adverse reactions, SJS Stevens-Johnson syndrome, SSKI potassium iodide, TB total bilirubin, TEN toxic epidermal necrolysis, } \\
\text { TNF alpha tumor necrosis factor alpha, T1DM type } 1 \text { diabetes mellitus, TSH thyroid-stimulating hormone, ULN upper limit of normal } \\
\text { a(Methyl)prednisolone or equivalent }\end{array}$} \\
\hline
\end{tabular}

with or without IVIG (intravenous immunoglobulin) for severe or steroid-resistant cases of SCARs (severe cutaneous adverse reactions) including SJS (Stevens-Johnson syndrome), TEN (toxic epidermal necrolysis), acute generalized exanthematous pustulosis, and DRESS (drug reaction with eosinophilia and systemic syndrome)/DIHS (drug-induced hypersensitivity syndrome).

\section{Enterocolitis}

Furthermore, grade 3 or 4 enterocolitis is mainly documented in IO/IO combination approaches (antiCTLA-4 plus anti-PD-1 antibodies) illustrated in checkmate 063 in advanced melanoma in up to $13 \%$ of patients [14]. However, even the sole administration of ICIs results in severe colitis in fewer than $<1 \%$ of patients, as shown in checkmate 037 and checkmate 066 (both melanoma trials using nivolumab) [15] and checkmate 017 in SQ-NSCLC (nivolumab) [3] and mentioned in treatment with anti-CTLA-4 antibody. This correlates with long-lasting responses persisting even after discontinuation of IO therapy [16]. In the case of grade 3 or 4 ir-AEs early administration of infliximab $5-10 \mathrm{mg} / \mathrm{kg}$ must be weighed if there is no response. Therefore, screening laboratory work (like HIV, hepatitis A \& B, blood quantiferon for tuberculosis) must be routinely done in patients at high risk in order to be prepared to start infliximab, if and when necessary. Administration of vedolizumab (anti-integrin antibody) is an option in patients who are refractory to infliximab and/or contraindicated for TNF-alpha blockade. Nonsteroidal anti-inflammatory drugs (NSAIDs) are reported to be associated with an increase in ICI-induced enterocolitis [17].

\section{Hepatitis}

Hepatitis (grade 3: AST/ALT 5-20 $\times$ ULN and/or total bilirubin 3-10× ULN) needs a second immunosuppressive agent like mycophenolate mofetil $1 \mathrm{~g}$ bid, which should be started if no improvement is assessed after 3 days.

\section{Pneumonitis}

Worsening to grade 3 ir-AE always demands permanent discontinuation of ICI, escalated prednisone $1-2 \mathrm{mg} / \mathrm{kg} /$ day IV and, if no improvement is observed, the addition of infliximab, MMF or IVIG over 5 days or cyclophosphamide. In life-threatening grade 4 pneumonitis urgent intervention is needed with empirical antibiotics and bronchoscopy with $\mathrm{BAL} \pm$ transbronchial biopsy if there are any doubts concerning the diagnosis. Less severe pneumonitis (grades 1-2) can be treated with oral prednisone $1-2 \mathrm{mg} / \mathrm{kg} /$ day whenever the patient is clinically stable and eligible for regular outpatient visits.

\section{Endocrinopathies}

In many cases, endocrine dysfunction (hypothyroidism, hyperthyroidism, adrenal insufficiency, hypophysitis) can be adequately treated on a symptomatic basis except in patients with diabetes, where such treatment could trigger a life-threatening situation as T1DM (type 1 diabetes mellitus) with ketoacidosis demands a strict work-up and insulin substitution.

Cranial MRI (with or without contrast) is indicated for complaints associated with vision changes, severe 
headaches and multiple endocrine abnormalities (including alterations in diverse electrolytes) in order to exclude hypophysitis [4].

\section{Nervous system toxicities}

Neurologic disorders like myasthenia gravis, Guillain-Barré syndrome, peripheral neuropathy, autonomic neuropathy, aseptic meningitis, encephalitis and transverse myelitis are treated with $1-2 \mathrm{mg} / \mathrm{kg} /$ day prednisone (grade 3) and, if there is no steroid response, IVIGs or plasmaphereses are ultimately alternative options.

\section{Myocarditis}

Cardiovascular complications (myocarditis, pericarditis, arrhythmias, impaired ventricular function with heart failure and vasculitis) require primary administration of corticosteroids. Furthermore, if grading deteriorates (grade 3 or 4) and/or there is no response to corticosteroid, treatment must be adapted by adding mycophenolate mofetil, infliximab or ATG (antithymocyte globulin).

\section{Future aspects and remarks}

In the context of ir-AEs, which vary in grading and duration of occurrence, manifestation always correlates with progression-free survival (documented in ongoing and already completed IO studies). Taking into account the fact that responders are primarily long responders, oncologists should maintain a sound awareness for the possibility of evolving immune-related adverse events of diverse grading [10] in order to be able to react promptly and correctly to keep their patients on ICI therapy as long as possible. Therefore, as so often in life, good timing can make all the difference.

Funding Open access funding provided by University of Innsbruck and Medical University of Innsbruck.

Conflict of interest J.D. Rudzki declares that he has received honoraria and speaker's fee from BMS, Roche, MSD, AstraZeneca, Amgen and served as advisor for BMS, Roche, MSD, AstraZeneca, Amgen.

Open Access This article is distributed under the terms of the Creative Commons Attribution 4.0 International License (http://creativecommons.org/licenses/by/4.0/), which permits unrestricted use, distribution, and reproduction in any medium, provided you give appropriate credit to the original author(s) and the source, provide a link to the Creative Commons license, and indicate if changes were made.

\section{References}

1. Hodi FS, O'Day SJ, McDermott DF, Weber RW, Sosman JA, Haanen JB, et al. Improved survival with ipilimumab in patients with metastatic melanoma. $\mathrm{N}$ Engl J Med. 2010;363:711-23.
2. Borghaei H, Paz-Ares L, Horn L, Spigel DR, Steins M, Ready NE, et al. Nivolumab versus docetaxel in advanced nonsquamous non-small-cell lung cancer. N Engl J Med. 2015;373:1627-39.

3. Brahmer J, Reckamp KL, Baas P, Crinò L, Eberhardt WEE, Poddubskaya E, et al. Nivolumab versus docetaxel in advanced squamous-cell non-small-cell lung cancer. N Engl J Med. 2015;373:123-35.

4. Herbst RS, Baas P, Kim D-W, Felip E, Perez-Gracia JL, Han $\mathrm{J}-\mathrm{Y}$, et al. Pembrolizumab versus docetaxel for previously treated,PD-L1-positive, advancednon-small-celllung cancer (KEYNOTE-010): a randomised controlled trial. Lancet. 2016;387:1540-50.

5. Palla AR, Doll D. Immunotherapy in Merkel cell carcinoma: role of avelumab. Immunotargets Ther. 2018;7:15-9.

6. Rittmeyer A, Barlesi F, Waterkamp D, Park K, Ciardiello F, von Pawel J, et al. Atezolizumab versus docetaxel in patients with previously treated non-small-cell lung cancer (OAK): a phase 3, open-label, multicentre randomised controlled trial. Lancet. 2017;389:255-65.

7. Antonia SJ, Villegas A, Daniel D, Vicente D, Murakami S, Hui R, et al. Durvalumab after chemoradiotherapy in stage III non-small-cell lung cancer. N Engl J Med. 2017;377:1919-29.

8. Weber JS, Kähler KC, Hauschild A. Management of immune-related adverse events and kinetics of response with ipilimumab. JClin Oncol. 2012;30:2691-7.

9. Zarogoulidis P, Chinelis P, Athanasiadou A, Tsiouda T, Trakada G, Kallianos A, et al. Possible adverse effects of immunotherapy in non-small cell lung cancer; treatment and follow-up of three cases. Respir Med Case Rep. 2017;22:101-5.

10. Brahmer JR, Lacchetti C, Schneider BJ, Atkins MB, Brassil $\mathrm{KJ}$, Caterino JM, et al. Management of immune-related adverse events in patients treated with immune checkpoint inhibitor therapy: American Society of Clinical Oncology clinical practice guideline. J Clin Oncol. 2018; https://doi. org/10.1200/JCO.2017.77.6385.

11. Postow MA. Managing immune checkpoint-blocking antibody side effects. Am Soc Clin Oncol Educ Book. 2015;35:76-83.

12. Puzanov I, Diab A, Abdallah K, Bingham CO, Brogdon C, Dadu R, et al. Managing toxicities associated with immune checkpoint inhibitors: consensus recommendations from the Society for Immunotherapy of Cancer (SITC) Toxicity Management Working Group. J Immunother Cancer. 2017; 5:95.

13. Johnson DB, Sullivan RJ, Menzies AM. Immune checkpoint inhibitors in challenging populations. Cancer. 2017;123:1904-11.

14. Weber JS, Gibney G, Sullivan RJ, Sosman JA, Slingluff CL, Lawrence DP, etal. Sequential administration of nivolumab and ipilimumab with a planned switch in patients with advanced melanoma (CheckMate 064): an open-label, randomised, phase 2 trial. Lancet Oncol. 2016;17:943-55.

15. Weber JS, Postow M, Lao CD, Schadendorf D. Management of adverse events following treatment with antiprogrammed death-1 agents. Oncologist. 2016;21:1230-40.

16. Beck KE, Blansfield JA, Tran KQ, Feldman AL, Hughes MS, Royal RE, et al. Enterocolitis in patients with cancer after antibody blockade of cytotoxic T-lymphocyte-associated antigen 4. JClin Oncol. 2006;24:2283-9.

17. Marthey L, Mateus C, Mussini C, Nachury M, Nancey S, Grange F, et al. Cancer immunotherapy with anti-CTLA4 monoclonal antibodies induces an inflammatory bowel disease. JCrohns Colitis. 2016;10:395-401. 